\title{
Myocardial bridging is an independent predictor of positive spasm provocation testing by intracoronary ergonovine injections: a retrospective observational study
}

\author{
Riku Arai ${ }^{1}$ (1) Hiroto Kano ${ }^{1}$. Shinya Suzuki ${ }^{1}$ Hiroaki Semba ${ }^{1} \cdot$ Takuto Arita $^{1} \cdot$ Naoharu Yagi $^{1} \cdot$ Takayuki Otsuka $^{1}$. \\ Shunsuke Matsuno ${ }^{1}$. Minoru Matsuhama ${ }^{1}$. Yuko Kato ${ }^{1}$. Tokuhisa Uejima ${ }^{1} \cdot$ Yuji Oikawa $^{1}$ - Yasuo Okumura ${ }^{2}$. \\ Junji Yajima ${ }^{1} \cdot$ Takeshi Yamashita $^{1}$
}

Received: 16 July 2019 / Accepted: 20 September 2019 / Published online: 27 September 2019

(c) The Author(s) 2019

\begin{abstract}
The relationship between myocardial bridging (MB) and coronary spasms during spasm provocation testing (SPT) remains unclear. We aimed to investigate whether MB was correlated with the SPT by ergonovine (ER) injections in a retrospective observational study. Of the 3340 patients who underwent a first coronary angiography, 166 underwent SPT using ER injections and were divided into 2 groups: $\mathrm{MB}(+)(n=23)$, and $\mathrm{MB}(-)(n=143)$. $\mathrm{MB}$ was defined as an angiographic reduction in the diameter of the coronary artery during systole. The patients who had severe organic stenosis in the left anterior descending coronary artery were excluded. The $\mathrm{MB}(+)$ group more frequently had diabetes mellitus and chronic kidney disease, and a thicker interventricular septum thickness. The rate of SPT-positivity was higher in the MB(+) group than MB(-) group (56.5\% vs. $22.4 \%, P=0.001)$. A multivariate regression analysis showed that the presence of $\mathrm{MB}$ was independently associated with SPT-positivity (odds ratio $5.587,95 \%$ confidence interval $2.061-15.149, P=0.001$ ). In conclusion, coronary spasms during provocation tests with ER independently correlated with the MB. MB may predict coronary spasms.
\end{abstract}

Keywords Myocardial bridging $\cdot$ Spasm provocation test $\cdot$ Ergonovine $\cdot$ Coronary spasm

\section{Introduction}

Myocardial bridging (MB) is an anomaly characterized by epicardial coronary vessels diving into the myocardium. MB is reflected as "squeezing" in coronary angiography (CAG). The frequency of MB has been reported in $0.5-12 \%$ of CAG images [1, 2]. Although MB has been considered as a benign condition, several complications have been reported, including ischemic chest pain [3], acute coronary syndrome (ACS) [4], sudden cardiac death [5], syncope [6], and coronary spasms [7]. Several reports have suggested that MB increases the risk of coronary spasms, especially in the MB

Riku Arai

riku.arai@icloud.com

1 Department of Cardiovascular Medicine, The Cardiovascular Institute, Nishiazabu 3-2-19, Minato-ku, Tokyo 1060031, Japan

2 Division of Cardiology, Department of Medicine, Nihon University School of Medicine, Tokyo, Japan segments because the longstanding compression-relaxation effects of MB may induce focal endothelial dysfunction by direct stress on the endothelium with enhanced vasoreactivity [7-10]. However, Sara et al. reported that MB was significantly associated with not only focal (epicardial) but also microvascular endothelial dysfunction in patients with nonobstructive coronary arteries [11]. Nevertheless, although there is a pathophysiological relationship between MB and endothelial dysfunction, little is known as to whether MB is associated with coronary spasms during spasm provocation testing (SPT) [12]. Therefore, we studied whether MB was related to coronary spasms by SPT using intracoronary ergonovine (ER) injections in a retrospective observational study. 


\section{Methods}

\section{Study participants and protocol}

Between January 2011 and March 2017, 7930 patients underwent CAG at the Cardiovascular Institute Hospital in Tokyo, Japan. The flowchart for the patient selection is shown in Fig. 1. The first set of exclusion criteria were as follows: (1) severe stenosis (as subtotal or total occlusion) in the left anterior descending coronary artery (LAD), in which MB was mostly involved [1] and (2) CAG more than once in this hospital, i.e., this was not the first CAG. Then, 3340 patients who underwent a first CAG without any severe organic stenosis in the LAD were enrolled. To investigate the relationship between MB and coronary spasms, we selected 170 patients who underwent SPT by intracoronary ER injections. We first evaluated how to select the patients who underwent SPT (SPT group). Among the 3340 patients who had a first CAG without any severe stenosis in the LAD, we compared the patient characteristics between the 170 SPT patients and remaining 3170 patients who did not undergo SPT (non-SPT group). The second set of exclusion criteria was as follows: (1) a history of percutaneous coronary intervention (PCI) and (2) acetylcholine ( $\mathrm{ACH})$ used in the SPT. Finally, a total of 166 patients were retrospectively analysed. The presence of MB was defined as an angiographic reduction in the diameter of the coronary artery during systole, also known as the "milking effect"' between systole and diastole [13].

The study protocol complied with the Declaration of Helsinki. Written informed consent for the CAG and SPT was obtained from all patients and the ethics committee at the Cardiovascular Institute approved the study protocol.

\section{Data collection on admission for the first CAG}

For each patient, the cardiovascular status was evaluated using echocardiography and serum tests on admission for the first CAG. The cardiovascular risk factors were defined as follows: hypertension (use of antihypertensive agents, systolic blood pressure $\geq 140 \mathrm{mmHg}$, or diastolic blood pressure $\geq 90 \mathrm{mmHg}$ ), diabetes mellitus (use of oral hypoglycaemic agents or insulin, or glycosylated hemoglobin $\geq 6.5 \%$ ), dyslipidemia (use of statins or drugs for lowering triglyceride, low-density lipoprotein $\geq 140 \mathrm{mg} / \mathrm{dl}$, high-density lipoprotein $<40 \mathrm{mg} / \mathrm{dl}$, or triglyceride $\geq 150 \mathrm{mg} / \mathrm{dl}$ ), and chronic kidney disease [estimated glomerular filtration rate $\left.(\mathrm{eGFR})<60 \mathrm{ml} / \mathrm{min} / \mathrm{m}^{2}\right]$. Information regarding the medications included the use of antihypertensive agents, beta blockers, calcium channel blockers, renin-angiotensin system inhibitors, antiplatelet medications, and anticoagulants. The eGFR was

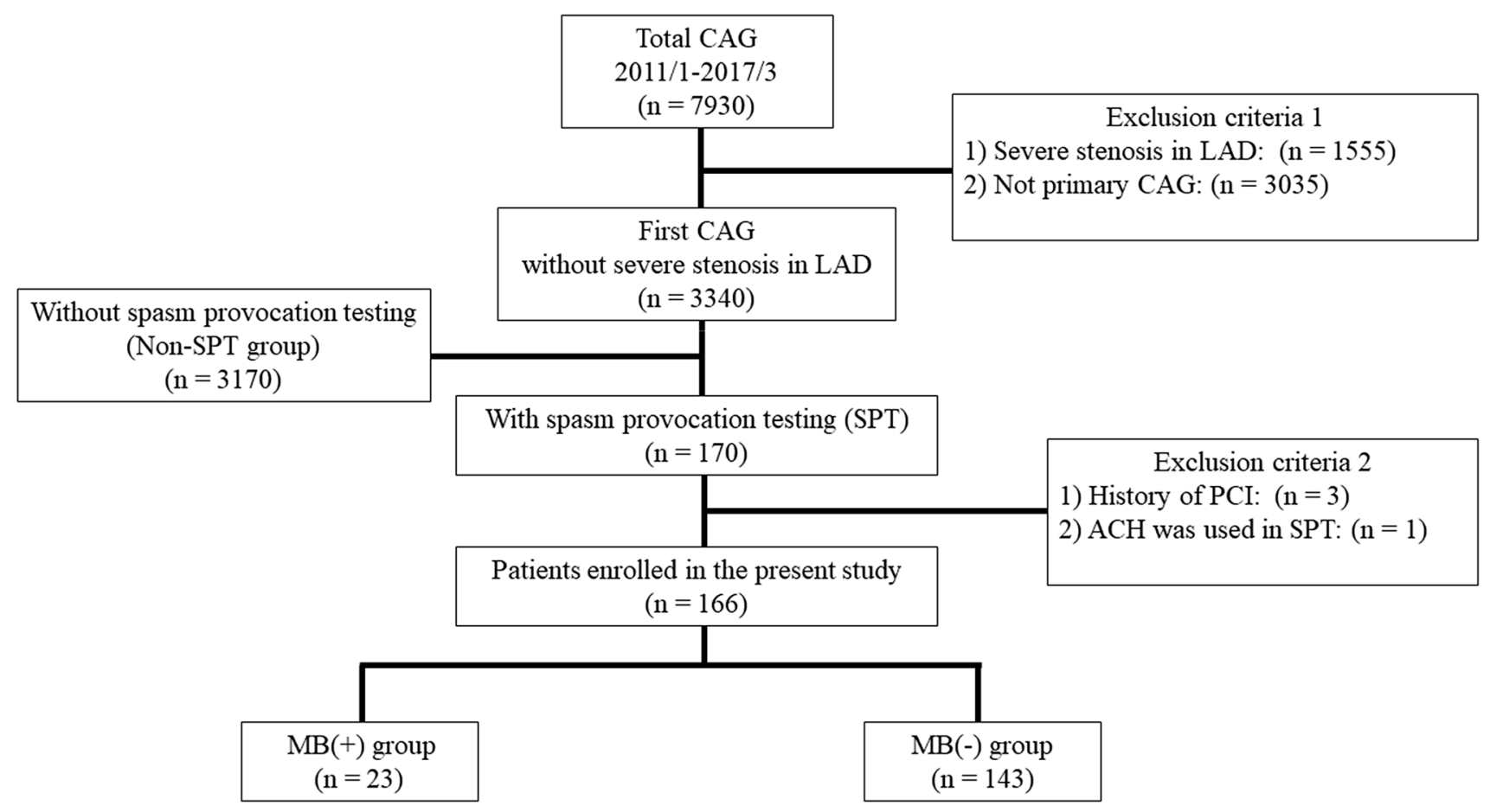

Fig. 1 Flowchart for the patient selection. $C A G$ coronary angiography, SPT spasm provocation test, $L A D$ left anterior descending coronary artery, $P C I$ percutaneous coronary intervention, $M B$ myocardial bridging, $A C H$ acetylcholine 
calculated using the equation for the Japanese population: $\mathrm{eGFR}=194 \times(\text { serum creatinine })^{-1.094} \times(\text { age })^{-0.287} \times(0.739$ for female patients).

\section{Spasm provocation test (SPT) using intracoronary ergonovine injections}

Experienced cardiologists performed the CAG via a transradial or transfemoral approach based on the current guidelines. Patients were instructed to stop using vasodilators, including calcium channel blockers and nitrates at least $24 \mathrm{~h}$ prior to the CAG and SPT. Diagnostic coronary angiograms were obtained of the right coronary artery (RCA) first, and then, of the left coronary artery (LCA). If coronary lesions with organic stenosis [defined as $>75 \%$ luminal narrowing according to the American College of Cardiology (ACC)/American Heart Association (AHA) classification [14]] were not observed, SPT was performed of the LCA and RCA using intracoronary ER injections. ER $(20 \mu \mathrm{g}$, $0.2 \mathrm{mg} / \mathrm{ml}$ ) in a $0.9 \%$ saline solution was injected up to 3 times per LCA and RCA. The SPT was first performed from the LCA because the catheter was still placed in the LCA after the diagnostic coronary angiogram. Standard 12-lead electrocardiograms (ECGs) were recorded carefully. At 1.5 min after each intracoronary injection of ER or when usual chest pain or significant ischemic ST changes on the electrocardiogram appeared, we obtained coronary angiograms. When the SPT of LCA was negative or symptoms and ECG changes improved rapidly even if the SPT of LCA was positive, SPT of RCA was continued. If symptoms and ECG changes persisted after the SPT of LCA, we injected nitrates intracoronary at that point and did not perform the SPT of RCA. After the SPT was completed, intracoronary nitrates were injected to reverse the coronary vasospasms. SPT-positivity was defined as a $>90 \%$ transient luminal narrowing, and typical chest symptoms, or significant ischemic ECG changes, according to the Japanese Circulation Society guidelines [15]. A positive criterion of $90 \%>$ stenosis was defined as severe stenosis with an angiographically visual contrast delay rather than quantitative coronary angiography (QCA) in the present study. SPT-negativity was defined as a case in which the positive criteria were not met even after 3 ER injections in both the LCA and RCA. The significant ischemic ECG changes were as follows: (1) ST-segment elevation of $>0.1 \mathrm{mV}$ in at least two contiguous leads, (2) ST-segment depression of $\geq 0.1 \mathrm{mV}$ in at least two contiguous leads, or (3) negative $U$ waves.

\section{Representative patients}

Representative patients are shown in Figs. 2, 3, 4, and 5.

\section{Statistical analysis}

All analyses were performed using SPSS version 19.0 software (SPSS Inc., Chicago, IL, USA). In all analyses, a $P<0.05$ was taken to indicate statistical significance.

The patients were divided into two groups according to the presence of $\mathrm{MB}[\mathrm{MB}(+)$ group; $n=23$, and $\mathrm{MB}(-)$ group; $n=143)$. The patient background was compared between the two groups. The differences in the categorical and consecutive variables were tested using the Chi-square and Student's $t$ tests, respectively. Then, univariate logistic regression analyses were conducted to identify the candidate variables among the clinical, laboratory, and echocardiography parameters. The multivariate model included the variables with a significance level of $P<0.005$ in the univariate analysis. We then performed a multivariate logistic regression analysis using stepwise methods.

\section{Results}

\section{Patient characteristics between the SPT group and non-SPT group}

The patient characteristics between the SPT group and nonSPT group are shown in Table 1. In the SPT group, the age was younger and comprised of males, cardiovascular risks, comorbidities, and organic heart disease were less prevalent than in the non-SPT group $(P<0.001$ for all). Among the 3170 patients in the non-SPT group, $220(6.9 \%)$ had MB in the mid-LAD, $18(0.6 \%)$ in the distal LAD, $1(0.03 \%)$ in the posterior descending branch of the RCA, and one each in the atrioventricular branch of the RCA and high lateral branch of the left circumflex coronary artery. Among the 23 (13.5\%) out of 170 SPT patients, all MBs were located in the mid-LAD.

\section{Patient characteristics between the patients with and without MB}

Table 2 summarizes the baseline clinical characteristics of the present study population. The prevalence of MB was $13.9 \%$ (23 of 166 patients). All patients had chest pain or chest discomfort before the CAG in each group. Compared to the patients without $\mathrm{MB}$, those with $\mathrm{MB}$ had higher prevalence rates of diabetes mellitus ( $26.1 \%$ vs. $9.8 \%, P=0.026)$ and chronic kidney disease $(60.9 \%$ vs. $35.7 \%, P=0.022)$. There were no significant differences in terms of the age, gender, smoking, drinking, prevalence of hypertension, dyslipidemia, hyperuricemia, laboratory data, medication at discharge, and echocardiographic data except for the interventricular septum thickness (IVST) $(10.2 \mathrm{~mm}$ vs. $9.3 \mathrm{~mm}$, $P=0.018$ ). 
Fig. 2 A representative case with MB and SPT-positivity in the LAD on the coronary angiogram at baseline (a-c), before (d, e), and after the SPT (f). At baseline and before the ER injections, the LCA angiogram shows no stenosis during the end-diastole phase $(\mathbf{a}, \mathbf{d})$ but MB is observed in the mid-LAD, as shown by squeezing (arrow heads) during the end-systole phase $(\mathbf{b}, \mathbf{e})$. The RCA angiogram shows no organic stenosis $(\mathbf{c})$. After the ER injections, the LAD becomes a total occlusion at the proximal lesion (f). After the ER injections into the LCA, the chest pain and ECG changes persist and nitrates are required to relieve the vasospasms, and therefore, SPT in the RCA was not performed. In this case, the culprit vessel of the SPTpositivity was the LAD, and the positional relationship of the vasospasms to the MB was the "segment proximal to the MB" (f). $C A G$ coronary angiography, $S P T$ spasm provocation test, $E R$ ergonovine, $L A D$ left anterior descending coronary artery, $R C A$ right coronary artery, $E C G$ electrocardiogram
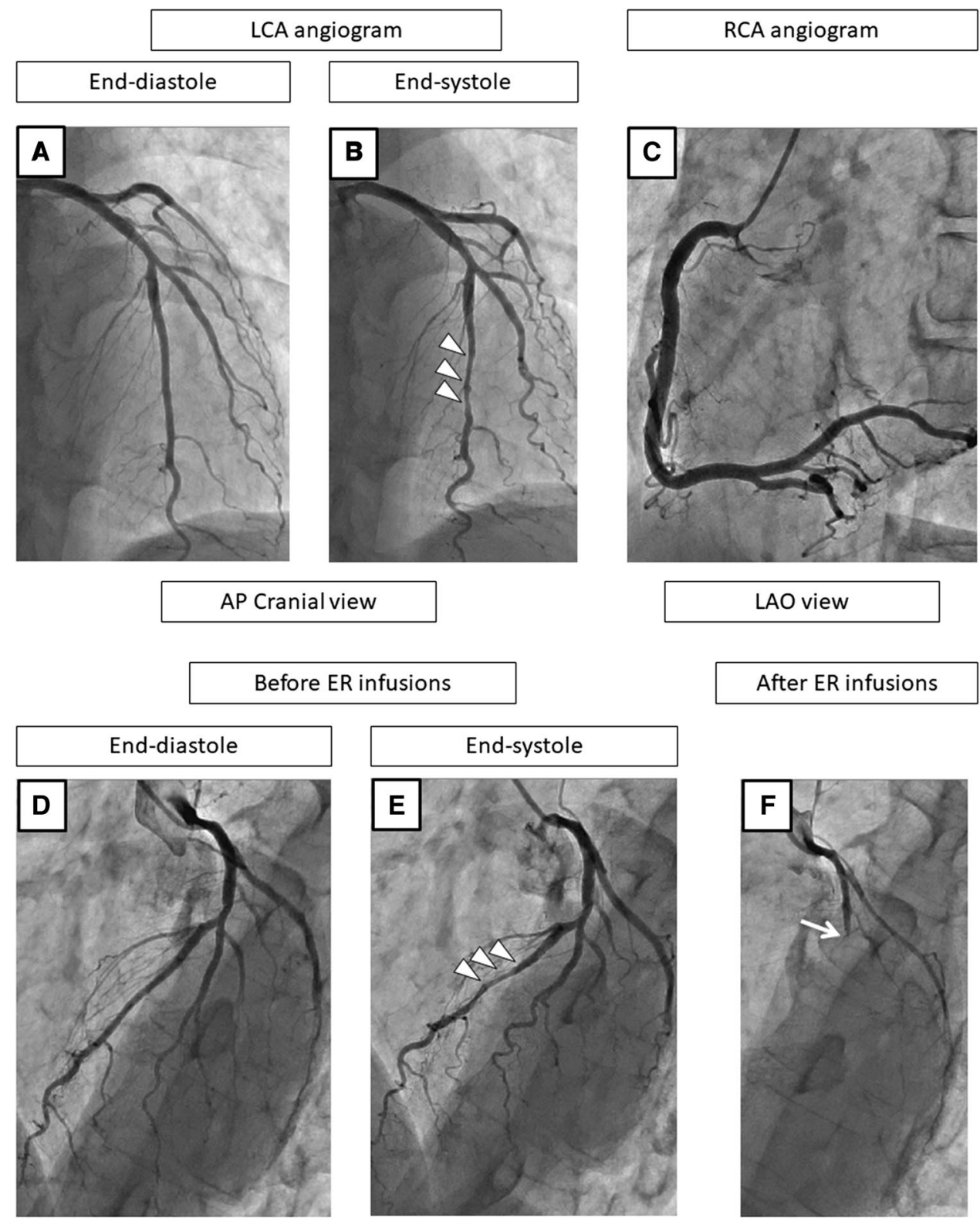

\section{After ER infusions}
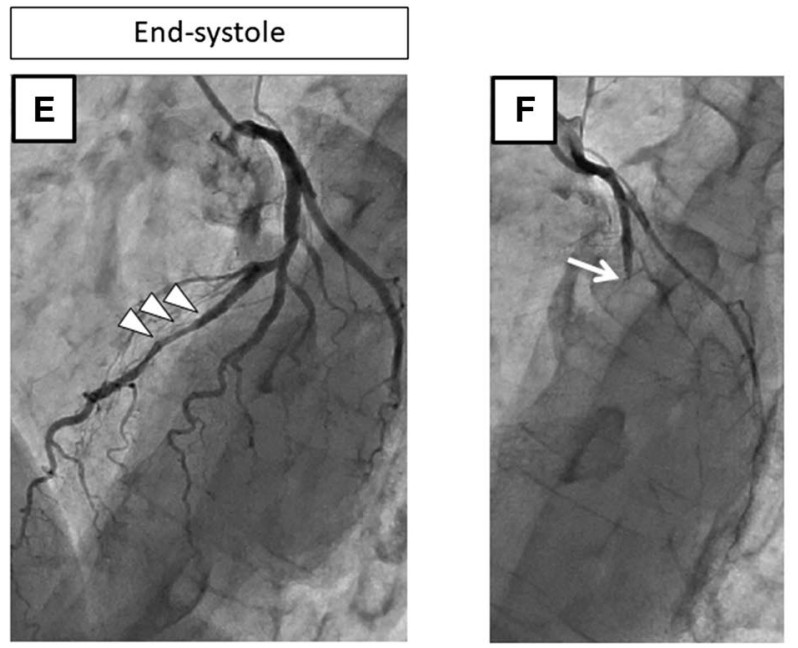

LAO Cranial view

\section{Association between the presence of MB and SPT}

The SPT-positivity rate was $27.1 \%$ (45 of 166 patients). The coronary artery features derived from the SPT between the patients with and without MB are summarized in Table 3. The patients with MB had higher SPT-positivity rates than those without $(56.5 \%$ vs $22.4 \%, P=0.001)$. In the MB (+) group, the most frequently recorded culprit vessel for SPTpositivity was the LAD [34.8\% (8/23 patients)], followed by the RCA [30.4\% (7/23)] and the LCX [4.3\% (1/23)]. In eight patients with SPT-positivity in the LAD, vasospasms occurred in diffuse lesions (i.e., proximal to the MB, on the $\mathrm{MB}$, and distal to the MB, 1 patient), the segment proximal to the MB (5 patients), on the MB (1 patients) in the LAD, and in the diagonal branch (1 patient), respectively. SPTpositivity was more frequently recorded in patients with $\mathrm{MB}$ in the LAD (34.8\% vs. $7.7 \%, P<0.001)$, and RCA (30.4\% vs. $11.2 \%, P=0.013$ ) than in those without (Table 3). Multivessel spasms also tended to be more often provoked in the $\mathrm{MB}(+)$ group $[8.7 \%(2 / 23)$ vs. $2.1 \%(3 / 143)$ in the MB (-) group, $P=0.086]$.

In the univariate logistic analysis, the presence of MB was significantly associated with SPT-positivity [odds ratio (OR) 4.509, 95\% confidence interval (CI) 1.809-11.241, $P=0.001]$. The male sex, prevalence of 


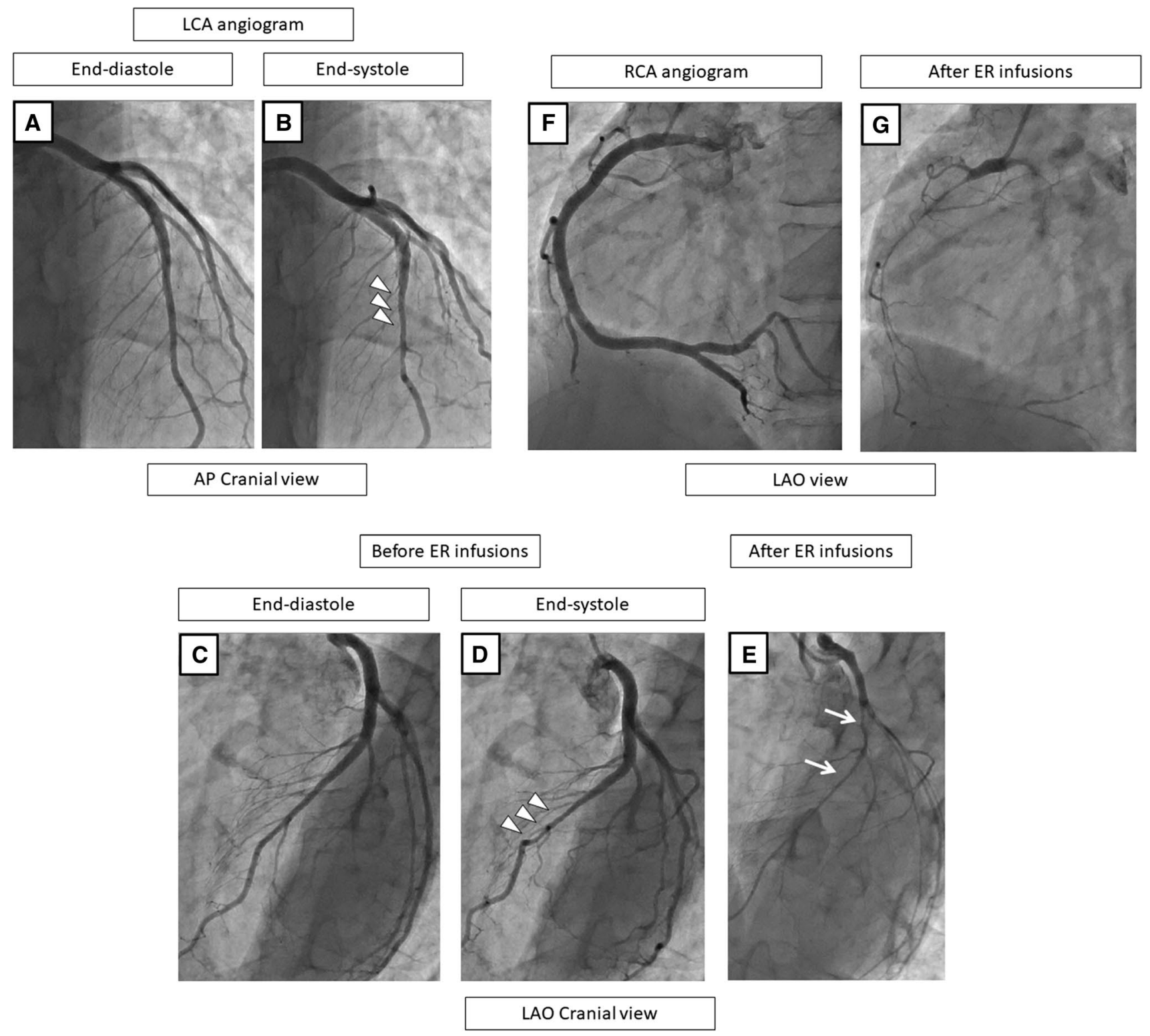

Fig. 3 A representative case with MB and a spastic vessel (SPT-negativity) in the LAD but with SPT-positivity in the RCA on the coronary angiogram at baseline $(\mathbf{a}, \mathbf{b})$, before $(\mathbf{c}, \mathbf{d}, \mathbf{f})$ and after the SPT $(\mathbf{e}, \mathbf{g})$. At baseline and before the ER injections, the LCA angiogram shows no organic stenosis during the end-diastole phase $(\mathbf{a}, \mathbf{c})$, but $\mathrm{MB}$ in the mid-LAD is observed as shown by the squeezing (arrow heads) during the end-systole phase (b, d). After the ER injections in the LCA, the LAD exhibits severe stenosis without a delay (e), but no ECG changes or chest symptoms occur. Before the ER injections into the RCA, no stenosis is observed (f), but the ER injections cause a subtotal occlusion (g). In this case, the culprit vessel of the SPTpositivity was the RCA, even though the LAD was a spastic vessel, suggesting a certain degree of endothelial dysfunction in the LAD. $C A G$ coronary angiography, $S P T$ spasm provocation test, ER ergonovine, $L A D$ left anterior descending coronary artery, $L C A$ left coronary artery, $R C A$ right coronary artery, $E C G$ electrocardiogram hypertension, chronic kidney disease, and hyperuricemia were also significantly associated with SPT-positivity $(P<0.005$, Table 4$)$. In multivariate logistic regression analysis, the presence of MB was an independent predictor of SPT-positivity (OR 5.587, 95\% CI 2.061-15.149, $P=0.001$ ), when adjusted for the covariables (Table 4).

\section{Discussion}

\section{Major findings}

The major findings of the present study were as follows: (1) in the patients with MB, the prevalence of diabetes 
Fig. 4 A representative case with MB and SPT-negativity in the LAD but with SPT-positivity in the RCA on the coronary angiogram before $(\mathbf{a}-\mathbf{c})$ and after the SPT (c, f). Before the ER injections, the LCA angiogram shows no significant stenosis during end-diastole (a) but MB in the mid-LAD is observed as shown by the "squeezing" (arrow heads) (b). After the ER injections into the LCA, the LAD does not have any spastic changes (c). Before the ER injection in the RCA, no stenosis was observed (e), but the ER injections cause a subtotal occlusion (f). In this case, the culprit vessel of the SPT-positivity was the RCA. $C A G$ coronary angiography, $S P T$ spasm provocation test, $E R$ ergonovine, $L C A$ left coronary artery, $L A D$ left anterior descending coronary artery, $R C A$ right coronary artery

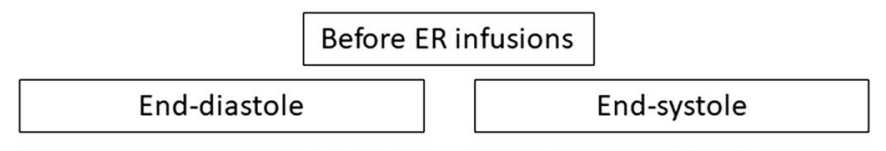

After ER infusions
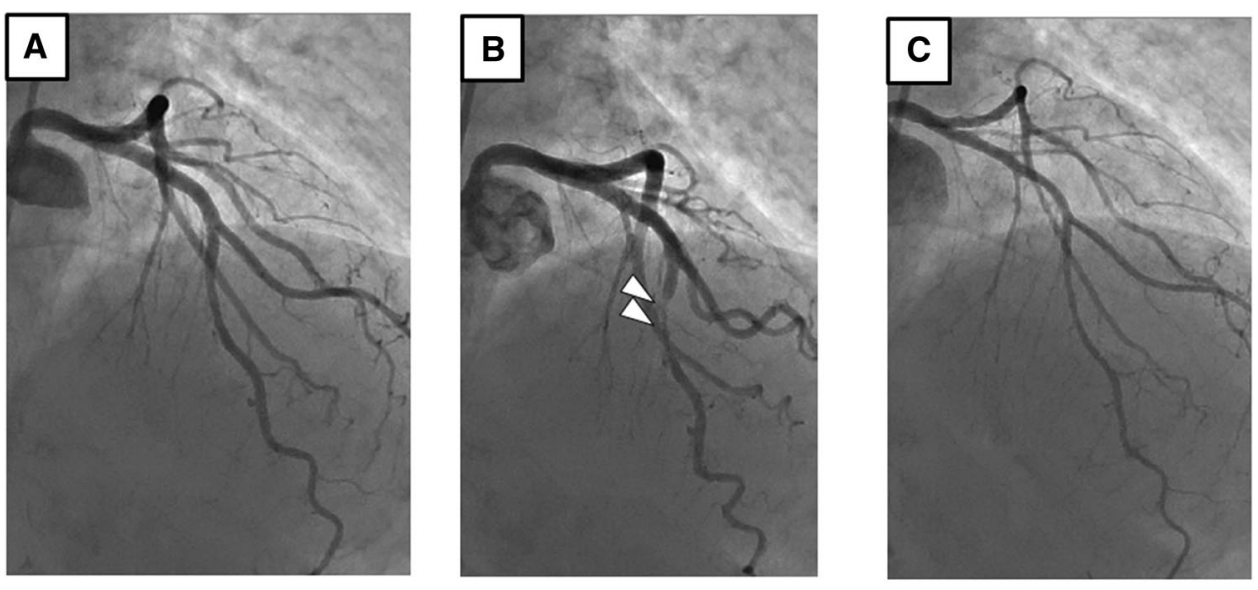

AP Cranial view

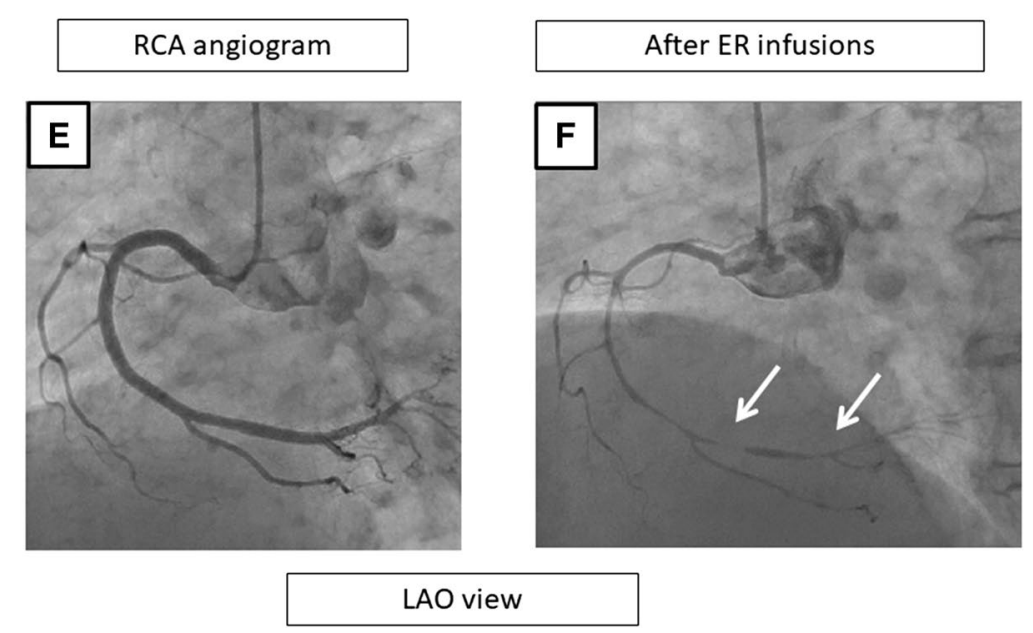

mellitus and chronic kidney disease was more frequent, and the IVST was thicker than in those without, (2) SPTpositivity by intracoronary ER injections was more frequent in patients with $\mathrm{MB}$ than in those without, especially of the LAD and RCA, (3) a multivariate logistic regression analysis showed that the presence of MB was an independent predictor of SPT-positivity by intracoronary ER injections, and (4) in patients with MB, the LAD was the most frequent culprit vessel of SPT-positivity, especially proximal to the MB in the LAD. To the best of our knowledge, this is the first report to suggest a clinical relationship between MB and SPT-positivity using intracoronary ER injections.

\section{Frequency and positivity rate of SPT compared with the previous reports}

The rate of SPT enforcement at our hospital was 170 cases in 7 years (between 2011 and 2017). According to a report examining SPT enforcement facilities in Japan [16], the number of SPT enforcements at our hospital was relatively high. In the specific population of patients who undergo intracoronary SPT, the prevalence of MB was $13.9 \%$ (23 of 166 patients) in our study. That rate was lower than that of the previous studies on SPT ranging from 31 to $36 \%$ [8, $12,17]$. The difference was evident in the SPT-positivity rate between our study and the previous studies (Table 5). 

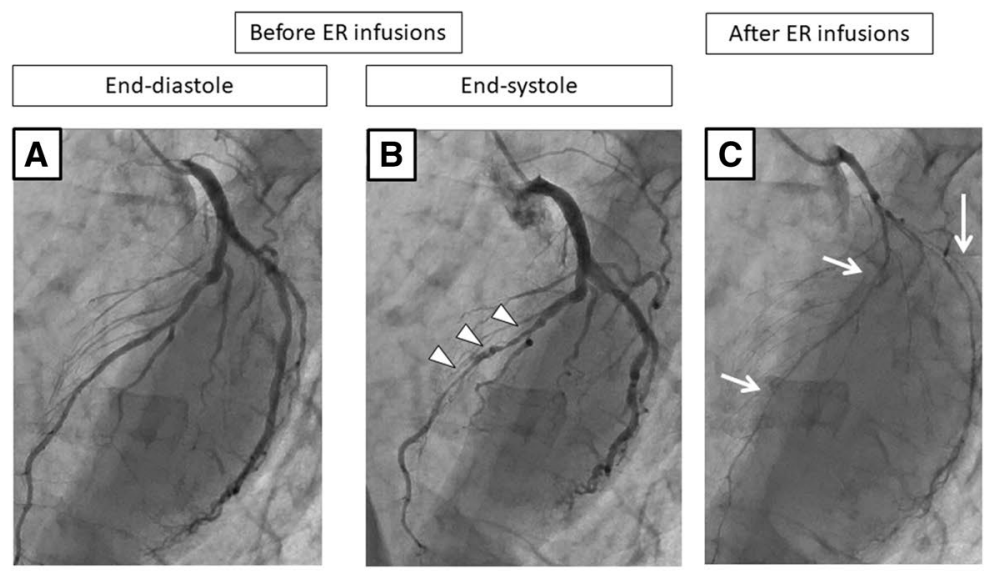

LAO Cranial view
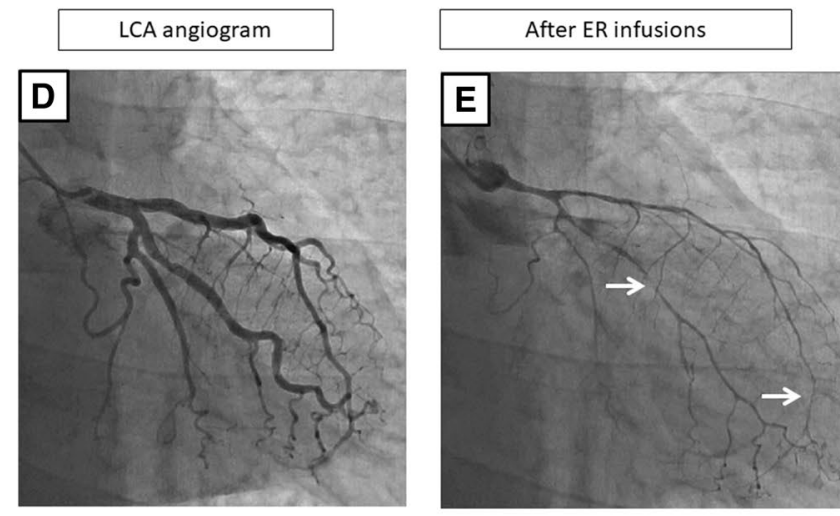

RAO Caudal view

Fig. 5 A representative case with diffuse MB in the LAD and multivessel spasms on the coronary angiogram before $(\mathbf{a}, \mathbf{b}, \mathbf{f})$ and after the SPT $(\mathbf{c}-\mathbf{e}, \mathbf{g})$. Before the ER injections, the LCA exhibits no stenosis during end-diastole (a), but MB in the mid-LAD is observed as shown by the "squeezing" (arrow head) (b). After the ER injections in the LCA, both the LAD and LCX show a subtotal occlusion with a delay $(\mathbf{c}, \mathbf{e})$, and chest pain and ECG changes occur. Thereafter, the chest pain and ECG changes improved without using nitrate infusions (d), so the SPT of the RCA was continued and no significant stenosis

The SPT-positivity rate in our study was $27.1 \%$, which was lower than that in the previous studies of $63.4 \%$ [17], $49.0 \%$ [12], and $51.8 \%$ [8], respectively. In particular, the SPT-positivity rate in the patients without MB differed, i.e., $22.4 \%$ in our study vs. $53.4 \%$ [17], $43.3 \%$ [12], and $39.7 \%$ [8], respectively, in the previous studies. There were several possible reasons for that difference. First, our hospital specializes in cardiovascular diseases. Our SPT-positivity rate was low because SPT was performed more frequently than in the other general hospitals [16]. The second reason was the different definitions of SPT-positivity between our study and that of the previous reports. Teragawa et al. reported that SPT-positivity was defined as a $\geq 50 \%$ reduction in the arterial diameter after ACH and/or ER [8]. In contrast, the

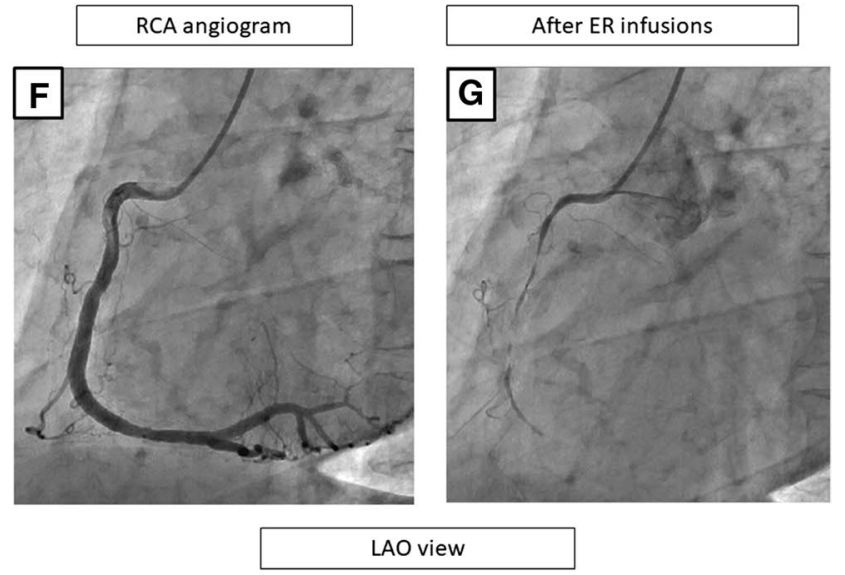

was observed (f). The ER injections in the RCA cause a total occlusion (g). In this case, the culprit vessels of the SPT-positivity were the RCA, LAD, and LCX. The positional relationship of the vasospasms to the MB segment was "diffuse, namely the segments proximal to the MB, on the MB, and distal to the MB" (c, e). CAG coronary angiography, $S P T$ spasm provocation test, $E R$ ergonovine, $L C A$ left coronary artery, $L A D$ left anterior descending coronary artery, $R C A$ right coronary artery, $L C X$ left circumflex artery, $E C G$ electrocardiogram

outcome of the present study was SPT-positivity as suggested by a guideline [15], namely the total or subtotal occlusion of the coronary artery with symptoms and/or significant ECG changes. Moderate-to-severe stenotic changes (50-90\%) after provocation tests were not included as the SPT-positive culprit vessel in the present study. The third reason was the difference in the provocation drugs between our study and that of the previous reports. ACH and ER have been employed in SPT. While ACH was infused to evaluate the endothelial function in the previous reports $[11,12]$, we used ER. There has not been a definite statement as to which drugs should be infused for SPT according to our guidelines. Furthermore, because the amount of the ER injections in the present study tended to less than previously reported [18], 
Table 1 Baseline characteristics between the patients who underwent an SPT (SPT group) and those who did not undergo an SPT (non-SPT group)

\begin{tabular}{|c|c|c|c|}
\hline & SPT group $(n=170)$ & $\begin{array}{l}\text { Non-SPT group } \\
(n=3170)\end{array}$ & $P$ value \\
\hline Age (year) & $59.3 \pm 11.5$ & $65.3 \pm 11.1$ & $<0.001$ \\
\hline Male sex & $109(64.1 \%)$ & $2409(76.0 \%)$ & $<0.001$ \\
\hline Body mass index $\left(\mathrm{kg} / \mathrm{m}^{2}\right)$ & $23.8 \pm 3.5$ & $24.3 \pm 3.8$ & 0.078 \\
\hline Smoking & $81(47.6 \%)$ & $1567(49.4 \%)$ & 0.650 \\
\hline Drinking & $94(55.3 \%)$ & $1558(49.1 \%)$ & 0.118 \\
\hline Hypertension & $84(49.5 \%)$ & $2167(68.4 \%)$ & $<0.001$ \\
\hline Dyslipidemia & $68(40.0 \%)$ & $1875(59.1 \%)$ & $<0.001$ \\
\hline Diabetes mellitus & $22(12.9 \%)$ & $1085(34.2 \%)$ & $<0.001$ \\
\hline Hyperuricemia & $28(16.5 \%)$ & $1035(32.6 \%)$ & $<0.001$ \\
\hline Chronic kidney disease & $66(38.8 \%)$ & $2096(66.1 \%)$ & $<0.001$ \\
\hline History of PCI & $3(1.8 \%)$ & $307(9.7 \%)$ & $<0.001$ \\
\hline History of CABG & 0 & $33(1.0 \%)$ & 0.181 \\
\hline Atrial fibrillation & $9(5.3 \%)$ & $289(9.1 \%)$ & 0.089 \\
\hline NYHA $\geq$ II & $4(2.4 \%)$ & $880(27.8 \%)$ & $<0.001$ \\
\hline Organic heart disease & $23(13.5 \%)$ & $2402(75.8 \%)$ & $<0.001$ \\
\hline Ischemic heart disease & $13(7.6 \%)$ & $1864(58.8 \%)$ & $<0.001$ \\
\hline Valvular disease & $8(4.7 \%)$ & $543(17.1 \%)$ & $<0.001$ \\
\hline Cardiomyopathy & $2(1.2 \%)$ & $337(10.6 \%)$ & $<0.001$ \\
\hline Congenital heart disease & $1(0.6 \%)$ & $20(0.6 \%)$ & 0.945 \\
\hline \multicolumn{4}{|l|}{ Laboratory data } \\
\hline Creatinine (mg/dl) & $0.8 \pm 0.2$ & $1.1 \pm 1.3$ & 0.001 \\
\hline eGFR (ml/min/1.73 m²) & $65.1 \pm 16.5$ & $53.1 \pm 17.6$ & $<0.001$ \\
\hline Uric acid (mg/dl) & $5.4 \pm 1.2$ & $5.8 \pm 1.5$ & 0.001 \\
\hline LDL cholesterol (mg/dl) & $112.1 \pm 29.9$ & $109.8 \pm 32.2$ & 0.398 \\
\hline HbA1c (NGSP) (\%) & $5.8 \pm 0.7$ & $6.2 \pm 1.0$ & $<0.001$ \\
\hline \multicolumn{4}{|l|}{ Medication at discharge } \\
\hline Antihypertensive agents & $76(44.7 \%)$ & $2170(68.5 \%)$ & $<0.001$ \\
\hline Beta blockers & $15(8.8 \%)$ & $1081(34.1 \%)$ & $<0.001$ \\
\hline Calcium channel blockers & $84(49.4 \%)$ & $1351(42.6 \%)$ & 0.081 \\
\hline Renin-angiotensin system inhibitors & $28(16.5 \%)$ & $1476(46.6 \%)$ & $<0.001$ \\
\hline Antiplatelets & $37(21.8 \%)$ & $1662(52.4 \%)$ & $<0.001$ \\
\hline Anticoagulants & $7(4.1 \%)$ & $430(13.6 \%)$ & $<0.001$ \\
\hline Diuretics & $1(0.6 \%)$ & $294(9.3 \%)$ & $<0.001$ \\
\hline \multicolumn{4}{|l|}{ Echocardiographic data } \\
\hline Interventricular septum thickness (mm) & $9.5 \pm 1.6$ & $10.5 \pm 2.1$ & $<0.001$ \\
\hline Left ventricular diameter at end- diastole $(\mathrm{mm})$ & $45.7 \pm 4.5$ & $47.7 \pm 7.0$ & $<0.001$ \\
\hline Left ventricular ejection fraction $(\%)$ & $68.5 \pm 6.4$ & $62.6 \pm 13.4$ & $<0.001$ \\
\hline Left atrial dimension $(\mathrm{mm})$ & $35.1 \pm 6.1$ & $38.2 \pm 7.4$ & $<0.001$ \\
\hline$E / e^{\prime}$ & $9.5 \pm 3.1$ & $12.4 \pm 5.4$ & 0.010 \\
\hline \multicolumn{4}{|l|}{ Location of MB } \\
\hline Proximal LAD & 0 & 0 & - \\
\hline Mid-LAD & $23(13.5 \%)$ & $220(6.9 \%)$ & 0.001 \\
\hline Distal LAD & 0 & $18(0.6 \%)$ & 0.325 \\
\hline Posterior descending branch (\#4PD) & 0 & $1(0.03 \%)$ & 0.817 \\
\hline Atrioventricular branch (\#4AV) & 0 & $1(0.03 \%)$ & 0.817 \\
\hline High lateral branch & 0 & $1(0.03 \%)$ & 0.817 \\
\hline
\end{tabular}

Data given as the mean $\pm \mathrm{SD}$ or $n(\%)$

$S P T$ spasm provocation test, $C A G$ coronary angiography, $L A D$ left anterior descending coronary artery, NYHA New York Heart Association, $M B$ myocardial bridging, PCI percutaneous coronary intervention, $C A B G$ coronary artery bypass graft, $e G F R$ estimated glomerular filtration rate 
Table 2 Baseline characteristics of the study patients according to the presence of MB

\begin{tabular}{|c|c|c|c|}
\hline & $\mathrm{MB}(+)$ group $(n=23)$ & MB (-) group $(n=143)$ & $P$ value \\
\hline Age (year) & $58.5 \pm 11.8$ & $59.3 \pm 11.5$ & 0.779 \\
\hline Male sex & $16(69.6 \%)$ & $89(62.2 \%)$ & 0.499 \\
\hline Symptoms (Chest pain or chest discomfort) & All & all & - \\
\hline Body mass index $\left(\mathrm{kg} / \mathrm{m}^{2}\right)$ & $23.9 \pm 4.3$ & $23.7 \pm 3.3$ & 0.794 \\
\hline Smoking & $10(43.5 \%)$ & $67(46.9 \%)$ & 0.763 \\
\hline Drinking & $10(43.5 \%)$ & $81(56.6 \%)$ & 0.239 \\
\hline Hypertension & $9(39.1 \%)$ & $72(50.3 \%)$ & 0.318 \\
\hline Dyslipidemia & $11(47.8 \%)$ & $54(37.8 \%)$ & 0.359 \\
\hline Diabetes mellitus & $6(26.1 \%)$ & $14(9.8 \%)$ & 0.026 \\
\hline Hyperuricemia & $5(21.7 \%)$ & $21(14.7 \%)$ & 0.388 \\
\hline Chronic kidney disease & $14(60.9 \%)$ & $51(35.7 \%)$ & 0.022 \\
\hline \multicolumn{4}{|l|}{ Laboratory data } \\
\hline Creatinine (mg/dl) & $0.8 \pm 0.2$ & $0.7 \pm 0.2$ & 0.432 \\
\hline eGFR (ml/min/1.73 m²) & $63.5 \pm 18.9$ & $65.3 \pm 16.3$ & 0.621 \\
\hline Uric acid $(\mathrm{mg} / \mathrm{dl})$ & $5.7 \pm 1.4$ & $5.4 \pm 1.2$ & 0.331 \\
\hline LDL cholesterol (mg/dl) & $112.8 \pm 27.5$ & $111.9 \pm 30.1$ & 0.900 \\
\hline HbA1c (NGSP) (\%) & $6.1 \pm 0.9$ & $5.8 \pm 0.6$ & 0.139 \\
\hline \multicolumn{4}{|l|}{ Medication at discharge } \\
\hline Antihypertensive agents & $10(43.5 \%)$ & $63(44.1 \%)$ & 0.959 \\
\hline Beta blockers & $2(8.7 \%)$ & $12(8.4 \%)$ & 0.961 \\
\hline Calcium channel blockers & $12(52.2 \%)$ & $69(48.3 \%)$ & 0.727 \\
\hline Renin-angiotensin system inhibitors & $4(17.4 \%)$ & $21(14.7 \%)$ & 0.736 \\
\hline Antiplatelets & $2(8.7 \%)$ & $33(23.1 \%)$ & 0.117 \\
\hline Anticoagulants & $1(4.3 \%)$ & $6(4.2 \%)$ & 0.973 \\
\hline \multicolumn{4}{|l|}{ Echocardiographic parameters } \\
\hline Interventricular septum thickness (mm) & $10.2 \pm 1.9$ & $9.3 \pm 1.5$ & 0.018 \\
\hline Left ventricular diameter at end-diastole (mm) & $45.1 \pm 4.9$ & $45.7 \pm 4.4$ & 0.625 \\
\hline Left ventricular ejection fraction (\%) & $70.9 \pm 7.7$ & $68.1 \pm 6.1$ & 0.066 \\
\hline Left atrial dimension (mm) & $36.1 \pm 5.9$ & $34.9 \pm 6.1$ & 0.421 \\
\hline$E / e^{\prime}$ & $8.3 \pm 1.5$ & $9.8 \pm 3.2$ & 0.378 \\
\hline
\end{tabular}

Data given as the mean $\pm \mathrm{SD}$ or $n(\%)$

$M B$ myocardial bridging, $e G F R$ estimated glomerular filtration rate

there might have been the possibility of false negatives in the present study.

\section{Frequency and characteristics of the patients with MB}

The prevalence of MB in the CAG in the present study was $7.3 \%$ (243 of 3340 patients), which kept with the results of the other studies that have shown an MB prevalence ranging from 0.5 to $12 \%[1,2]$ in the CAG. Similar to the previous reports in Japanese patients $[8,12], 166$ patients in the SPT group were relatively young (55-63 years) but there was no significant age-difference with regard to the prevalence of MB (58.5 vs. 59.3, $P=0.779)$. We found an interesting observation that the prevalence of diabetes mellitus and chronic kidney disease was higher, and the IVST thicker in the patients with MB than in those without. Diabetes mellitus and chronic kidney disease have been known to be associated with endothelial dysfunction $[19,20]$, and thus, those comorbidities might have interacted with the MB for coronary spasms. Nonetheless, the predictive values of those comorbidities for the SPT-positivity were not independent. Although it is speculative, a thicker IVST in the patients with MB might be related to endothelial dysfunction due to a stronger coronary compression in the MB segment, leading to an increase in the mechanical stimulation effect at the MB segment, as discussed below.

\section{Possible mechanisms between MB and coronary spasms}

In the present study, the SPT-positivity by intracoronary ER injections was more frequent in patients with MB than 
Table 3 Coronary artery features of the spasm provocation test (SPT) by an intracoronary ergonovine (ER) infusion according to the presence of $\mathrm{MB}$

\begin{tabular}{lllr}
\hline & MB (+) group $(n=23)$ & MB $(-)$ group $(n=143)$ & $P$ value \\
\hline $\begin{array}{l}\text { SPT-positivity } \\
\text { Signs of ischemia }\end{array}$ & $13(56.5 \%)$ & $32(22.4 \%)$ & 0.001 \\
ECG changes & $13(56.5 \%)$ & $32(22.4 \%)$ & 0.001 \\
Chest symptoms & $15(65.2 \%)$ & $42(29.4 \%)$ & 0.001 \\
Tested coronary arteries & & & \\
RCA & $17(73.9 \%)$ & $127(88.8 \%)$ & 0.050 \\
LCA & $23(100 \%)$ & $143(100 \%)$ & - \\
Both RCA and LCA & $17(73.9 \%)$ & $127(88.8 \%)$ & 0.050 \\
Culprit vessel of SPT-positivity & & 0.013 \\
RCA & $7(30.4 \%)$ & $16(11.2 \%)$ & $<0.001$ \\
LAD & $8(34.8 \%)$ & $11(7.7 \%)$ & 0.806 \\
LCX & $1(4.3 \%)$ & $8(5.6 \%)$ & 0.086 \\
Multivessel spasm & $2(8.7 \%)$ & $3(2.1 \%)$ & \\
\hline
\end{tabular}

Data given as the mean $\pm \mathrm{SD}$ or $n(\%)$

$M B$ myocardial bridging, $E C G$ electrocardiogram, $R C A$ right coronary artery, $L C A$ left coronary artery, $L A D$ left anterior descending coronary artery, $L C X$ left circumflex artery

\begin{tabular}{|c|c|c|c|c|c|c|}
\hline \multirow[t]{2}{*}{ Variable } & \multicolumn{3}{|c|}{ Univariate model } & \multicolumn{3}{|c|}{ Multivariate model $^{\mathrm{a}}$} \\
\hline & Odds ratio & $95 \%$ CI & $P$ value & Odds ratio & $95 \% \mathrm{CI}$ & $P$ value \\
\hline $\mathrm{MB}(+)$ & 4.509 & $1.809-11.241$ & 0.001 & 5.587 & $2.061-15.149$ & 0.001 \\
\hline Age & 1.001 & $0.972-1.032$ & 0.937 & & & \\
\hline Male & 3.605 & $1.549-8.387$ & 0.003 & 3.798 & $1.557-9.266$ & 0.003 \\
\hline Smoking & 1.659 & $0.832-3.305$ & 0.150 & & & \\
\hline Drinking & 1.516 & $0.753-3.054$ & 0.244 & & & \\
\hline Hypertension & 2.113 & $1.048-4.262$ & 0.037 & 2.637 & $1.214-5.729$ & 0.014 \\
\hline Diabetes mellitus & 1.530 & $0.568-4.120$ & 0.400 & & & \\
\hline Dyslipidemia & 1.191 & $0.594-2.390$ & 0.622 & & & \\
\hline Chronic kidney disease & 2.230 & $1.112-4.474$ & 0.024 & & & \\
\hline Hyperuricemia & 2.779 & $1.171-6.595$ & 0.020 & & & \\
\hline
\end{tabular}

$M B$ myocardial bridging, $C I$ confidence interval

${ }^{a}$ Adjusted for chronic kidney disease and hyperuricemia
Table 4 Logistic regression analysis for spasm provocation test (SPT)-positivity in those without. Even after a multivariate adjustment, the presence of $\mathrm{MB}$ remained to be an independent predictor of SPT-positivity. The reported causes of coronary spasms are abnormal responses of the autonomic nervous system [21], endothelial dysfunction [22], abnormal or hyper-reaction of vascular smooth muscles [23], inheritance [24], and the specific anatomy of the coronary arteries [9, 12]. In patients with $\mathrm{MB}$, the reasons why MB is associated with coronary spasms are as follows: (1) the mechanical stimulation effect of $\mathrm{MB}$ causes longstanding mechanical stress in MB segments, leading to focal endothelial dysfunction [8] and (2) a structural lumen obstruction in MB segments causes hemodynamic and wall shear stress (WSS) changes [25], leading to endothelial dysfunction [26, 27]. Coronary compression by $\mathrm{MB}$ results in a relatively low WSS proximal and distal to the MB segment and a high WSS in the MB segment.
Similar to the previous reports [7-9, 12], the culprit vessel that was SPT-positive was most frequently located in the LAD, especially proximal to the MB segment in the LAD [12], suggesting endothelial dysfunction of that artery.

In general, the mechanism of coronary spasms may not be derived from only one cause, rather there may be several factors. Ohyama et al. reported that perivascular components, including perivascular adipose tissue and adventitial vasa vasorum caused an increased activity of Rho-kinase, leading to coronary spasms [28, 29]. The epicardial adipose tissue surrounding the LAD has been reported to be more directly linked to cardiovascular events than EATs surrounding the LCX and RCA [30]. Masuda et al. reported that endothelial nitric oxide synthase and endothelin-1, reflecting the endothelial function, were significantly lower in MB segments [31]. The difference in the expression of 
Table 5 SPT-positivity rate and prevalence of MB compared with the previous reports

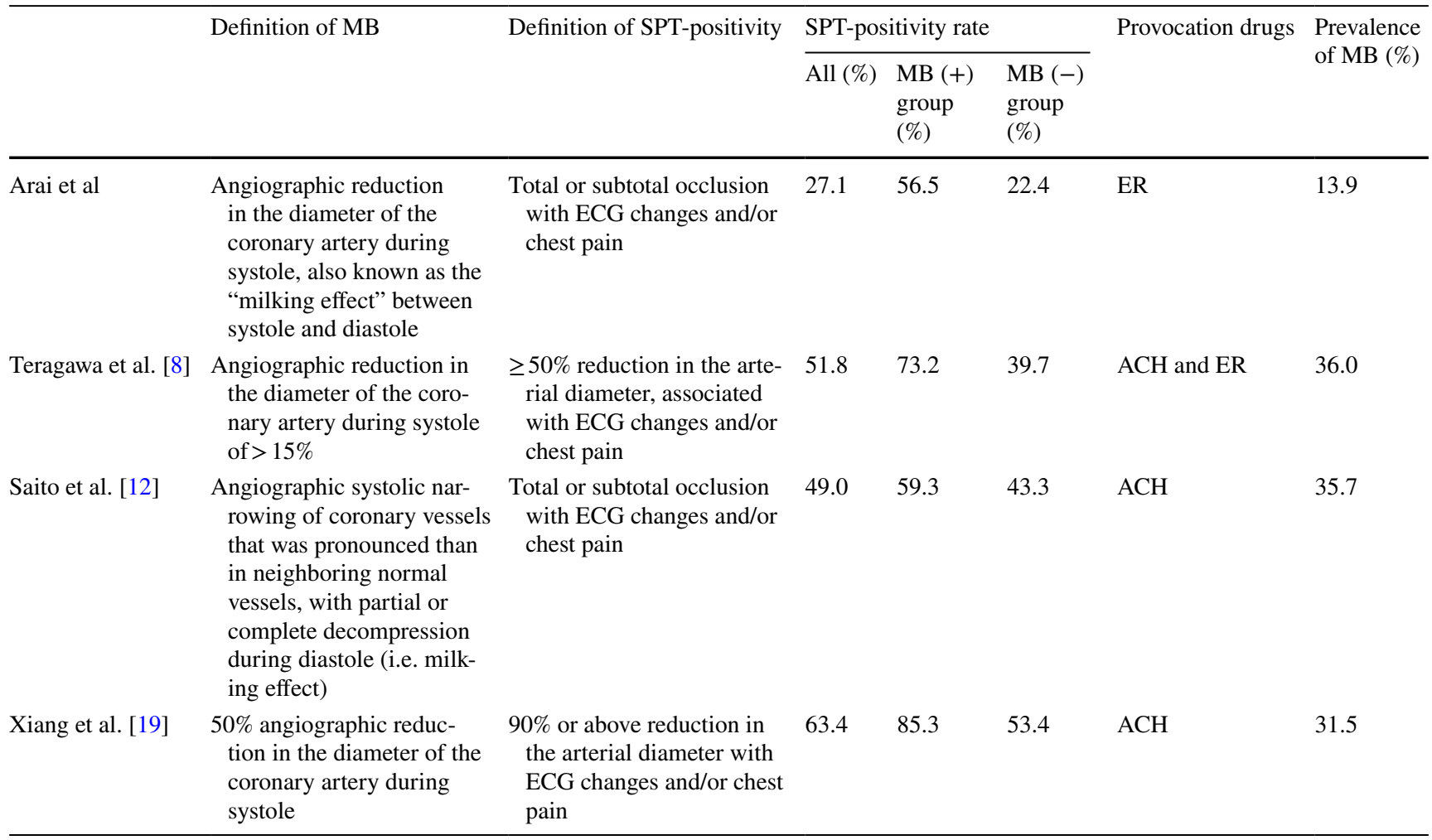

$S P T$ spasm provocation test, $M B$ myocardial bridging, $E C G$ electrocardiogram, $E R$ ergonovine, $A C H$ acetylcholine

these vasoactive agents in patients with MB may influence the microvascular function remotely, leading to not only focal (epicardial), but also microvascular endothelial dysfunction [11]. These mechanisms may cause that the culprit vessel of the SPT-positivity was recorded not only in MB segments in our study, even though the MB segments also tended to be spastic and suggested a certain degree of endothelial dysfunction in these patients (Fig. 3). Saito et al. reported that patients with MB had a higher SPT-positivity not only in the LAD (61\% vs. $39 \%, P<0.001)$ but also in the RCA (37\% vs. $25 \%, P=0.020)$ by intracoronary ACH testing [12], even using intracoronary ER testing, as we found in our study.This was also reinforced by our finding that multivessel spasms also tended to be more frequently provoked in the MB $(+)$ group than MB $(-)$ group $(P=0.086)$. The incidence of multivessel spasms in the MB $(+)$ group might have been further increased because ER provocation testing could not be performed in the RCA more frequently in this group $[26.1 \%(6 / 23)$ vs. $11.2 \%(16 / 143), P=0.050]$ when the LCA became SPT-positive with persistent ischemic ECG changes and/or chest pain that required nitrate injections to relieve the vasospasms.

\section{Limitations}

The present study had several limitations. First, this was a single-centre retrospective observational study, the sample size was rather small, and patient selection bias was present, which might have limited the generalizability of the present study. Nonetheless, a younger age and less comorbidities and cardiovascular risks in the patients who underwent SPT are generally well-known in routine clinical practice. Second, MB was defined by only the angiographic characteristics [32], and we did not perform other modalities, including coronary computed tomography angiography, positron emission tomography, or contrast stress echocardiography [33, 34]. Third, our analyses could not evaluate the length or depth of the MB segment, even though the hemodynamic impact of the MB depends on the thickness and length of the bridge [34]. Fourth, we could not perform SPT of the RCA in 6/13 cases among the patients with $\mathrm{MB}$ and in 16/32 cases among those without MB. Therefore, the number of SPT-positivity cases in the RCA might have been underestimated. Finally, we used only ER injections for the SPT, and the dose of the ER was lower than that used in the previous reports [18]. 


\section{Conclusion}

The presence of MB was an independent predictor of SPTpositivity in patients with chest pain without a significant obstruction of the coronary arteries. The patients who had MB on CAG might be carefully observed because coronary spasms could be the cause of their symptoms.

Acknowledgements We thank Shiro Ueda and Nobuko Ueda at Medical Edge Company Ltd., for assembling the database using the Clinical Study Supporting System, and Ineko Hayakawa, Hiroaki Arai, and Hirokazu Aoki for data management and system administration. We also thank Mr. John Martin for help with the English editing.

Funding This study was partially supported by the Practical Research Project for Lifestyle-Related Diseases including Cardiovascular Diseases and Diabetes Mellitus from the Japan Agency for Medical Research and Development, AMED (15656344).

\section{Compliance with ethical standards}

Conflict of interest Dr. Suzuki received research funding from Tanabe-Mitsubishi, and Daiichi-Sankyo. Dr. Takayuki Otsuka received research funding and remuneration from Nippon Boehringer Ingelheim. Dr. Yamashita received funding from Nippon Boehringer Ingelheim and Daiichi-Sankyo, and remuneration from Nippon Boehringer Ingelheim, Daiichi-Sankyo, Bayer Healthcare, Bristol-Myers Squibb, Pfizer, Eisai, and Ono Pharmaceutical.

Open Access This article is distributed under the terms of the Creative Commons Attribution 4.0 International License (http://creativeco mmons.org/licenses/by/4.0/), which permits unrestricted use, distribution, and reproduction in any medium, provided you give appropriate credit to the original author(s) and the source, provide a link to the Creative Commons license, and indicate if changes were made.

\section{References}

1. Tarantini G, Migliore F, Cademartiri F, Fraccaro C, Iliceto S (2016) Left anterior descending artery myocardial bridging: a clinical approach. J Am Coll Cardiol 68:2887-2899

2. Ishikawa Y, Kawawa Y, Kohda E, Shimada K, Ishii T (2011) Significance of the anatomical properties of a myocardial bridge in coronary heart disease. Circ J 75:1559-1566

3. Berry JF, von Mering GO, Schmalfuss C, Hill JA, Kerensky RA (2002) Systolic compression of the left anterior descending coronary artery: a case series, review of the literature, and therapeutic options including stenting. Catheter Cardiovasc Interv 56:58-63

4. Tauth J, Sullebarger T (1997) Myocardial infarction associated with myocardial bridging: case history and review of the literature. Catheter Cardiovasc Diagn 40:364-367

5. Cutler D, Wallace JM (1997) Myocardial bridging in a young patient with sudden death. Clin Cardiol 20:581-583

6. Ural E, Bildirici U, Celikyurt U, Kilic T, Sahin T, Acar E, Kahraman G, Ural D (2009) Long-term prognosis of non-interventionally followed patients with isolated myocardial bridge and severe systolic compression of the left anterior descending coronary artery. Clin Cardiol 32:454-457
7. Kodama K, Morioka N, Hara Y, Shigematsu Y, Hamada M, Hiwada K (1998) Coronary vasospasm at the site of myocardial bridge-report of two cases. Angiology 49:659-663

8. Teragawa H, Fukuda Y, Matsuda K, Hirao H, Higashi Y, Yamagata T, Oshima T, Matsuura H, Chayama K (2003) Myocardial bridging increases the risk of coronary spasm. Clin Cardiol 26:377-383

9. Kim JW, Seo HS, Na JO, Suh SY, Choi CU, Kim EJ, Rha SW, Park CG, Oh DJ (2008) Myocardial bridging is related to endothelial dysfunction but not to plaque as assessed by intracoronary ultrasound. Heart 94:765-769

10. Kim JW, Park CG, Suh SY, Choi CU, Kim EJ, Rha SW, Seo HS, Oh DJ (2007) Comparison of frequency of coronary spasm in Korean patients with versus without myocardial bridging. Am J Cardiol 100:1083-1086. https://doi.org/10.1016/j.amjca rd.2007.05.030

11. Sara JDS, Corban MT, Prasad M, Prasad A, Gulati R, Lerman LO, Lerman A (2019) The prevalence of myocardial bridging associated with coronary endothelial dysfunction in patients with chest pain and non-obstructive coronary artery disease. EuroIntervention. https://doi.org/10.4244/eij-d-18-00920

12. Saito Y, Kitahara H, Shoji T, Tokimasa S, Nakayama T, Sugimoto K, Fujimoto Y, Kobayashi Y (2017) Relation between severity of myocardial bridge and vasospasm. Int J Cardiol 248:34-38

13. Bourassa MG, Butnaru A, Lesperance J, Tardif JC (2003) Symptomatic myocardial bridges: overview of ischemic mechanisms and current diagnostic and treatment strategies. J Am Coll Cardiol 41:351-359

14. Scanlon PJ, Faxon DP, Audet AM, Carabello B, Dehmer GJ, Eagle KA, Legako RD, Leon DF, Murray JA, Nissen SE, Pepine CJ, Watson RM, Ritchie JL, Gibbons RJ, Cheitlin MD, Gardner TJ, Garson A Jr, Russell RO Jr, Ryan TJ, Smith SC Jr (1999) ACC/AHA guidelines for coronary angiography. A report of the American College of Cardiology/American Heart Association Task Force on practice guidelines (Committee on Coronary Angiography). Developed in collaboration with the Society for Cardiac Angiography and Interventions. J Am Coll Cardiol 33:1756-1824

15. (2014) Guidelines for diagnosis and treatment of patients with vasospastic angina (Coronary Spastic Angina) (JCS 2013). Circ J 78: 2779-2801

16. Sueda S, Oshita A, Izoe Y, Kohno H, Fukuda H (2006) Survey of coronary spasm in Japan before the establishment of spasm guidelines: analysis of questionnaires throughout Japan. J Cardiol 48:333-343

17. Xiang DC, Gong ZH, He JX, Ruan YJ, Xie ZH (2009) Characteristics of stress tests and symptoms in patients with myocardial bridge and coronary artery spasm. Coron Artery Dis 20:27-31

18. Sueda S, Kohno H (2018) Relationships between more than $90 \%$ coronary luminal narrowing induced by ergonovine provocation test and ECG ischemic change as well as chest symptoms. Heart Vessels 33:95-101

19. Koyama T, Tanaka A, Yoshida H, Oyama JI, Toyoda S, Sakuma M, Inoue T, Otsuka Y, Node K (2018) Comparison of the effects of linagliptin and voglibose on endothelial function in patients with type 2 diabetes and coronary artery disease: a prospective, randomized, pilot study (EFFORT). Heart Vessels 33:958-964

20. Tabata N, Hokimoto S, Akasaka T, Arima Y, Sakamoto K, Yamamoto E, Tsujita K, Izumiya Y, Yamamuro M, Kojima S, Kaikita K, Ogawa H (2016) Differential impact of peripheral endothelial dysfunction on subsequent cardiovascular events following percutaneous coronary intervention between chronic kidney disease (CKD) and non-CKD patients. Heart Vessels 31:1038-1044

21. Yasue H, Touyama M, Shimamoto M, Kato H, Tanaka S (1974) Role of autonomic nervous system in the pathogenesis of Prinzmetal's variant form of angina. Circulation 50:534-539 
22. Okumura K, Yasue H, Ishizaka H, Ogawa H, Fujii H, Yoshimura M (1992) Endothelium-dependent dilator response to substance $\mathrm{P}$ in patients with coronary spastic angina. J Am Coll Cardiol 20:838-844

23. Shimokawa H (2000) Cellular and molecular mechanisms of coronary artery spasm: lessons from animal models. Jpn Circ J 64:1-12

24. Yoshimura M, Yasue H, Nakayama M, Shimasaki Y, Ogawa H, Kugiyama K, Saito Y, Miyamoto Y, Ogawa Y, Kaneshige T, Hiramatsu H, Yoshioka T, Kamitani S, Teraoka H, Nakao K (2000) Genetic risk factors for coronary artery spasm: significance of endothelial nitric oxide synthase gene T-786-\%3eC and missense Glu298Asp variants. J Investig Med 48:367-374

25. Herrmann J, Higano ST, Lenon RJ, Rihal CS, Lerman A (2004) Myocardial bridging is associated with alteration in coronary vasoreactivity. Eur Heart J 25:2134-2142

26. Ortega J, Hartman J, Rodriguez J, Maitland D (2008) Post-treatment hemodynamics of a basilar aneurysm and bifurcation. Ann Biomed Eng 36:1531-1546

27. Fry DL (1969) Certain histological and chemical responses of the vascular interface to acutely induced mechanical stress in the aorta of the dog. Circ Res 24:93-108

28. Ohyama K, Matsumoto Y, Takanami K, Ota H, Nishimiya K, Sugisawa J, Tsuchiya S, Amamizu H, Uzuka H, Suda A, Shindo T, Kikuchi Y, Hao K, Tsuburaya R, Takahashi J, Miyata S, Sakata Y, Takase K, Shimokawa H (2018) Coronary adventitial and perivascular adipose tissue inflammation in patients with vasospastic angina. J Am Coll Cardiol 71:414-425

29. Ohyama K, Matsumoto Y, Nishimiya K, Hao K, Tsuburaya R, Ota H, Amamizu H, Uzuka H, Takahashi J, Ito K, Shimokawa H (2016) Increased coronary perivascular adipose tissue volume in patients with vasospastic angina. Circ J 80:1653-1656
30. Maimaituxun G, Shimabukuro M, Fukuda D, Yagi S, Hirata Y, Iwase T, Takao S, Matsuura T, Ise T, Kusunose K, Tobiume T, Yamaguchi K, Yamada H, Soeki T, Wakatsuki T, Harada M, Sata M (2018) Local thickness of epicardial adipose tissue surrounding the left anterior descending artery is a simple predictor of coronary artery disease-new prediction model in combination with framingham risk score. Circ J 82:1369-1378

31. Masuda T, Ishikawa Y, Akasaka Y, Itoh K, Kiguchi H, Ishii T (2001) The effect of myocardial bridging of the coronary artery on vasoactive agents and atherosclerosis localization. J Pathol 193:408-414

32. Rogers IS, Tremmel JA, Schnittger I (2017) Myocardial bridges: overview of diagnosis and management. Congenit Heart Dis 12:619-623

33. Mok S, Majdalany D, Pettersson GB (2019) Extensive unroofing of myocardial bridge: a case report and literature review. SAGE Open Med Case Rep. https://doi.org/10.1177/2050313x1882338 0

34. Corban MT, Hung OY, Eshtehardi P, Rasoul-Arzrumly E, McDaniel M, Mekonnen G, Timmins LH, Lutz J, Guyton RA, Samady H (2014) Myocardial bridging: contemporary understanding of pathophysiology with implications for diagnostic and therapeutic strategies. J Am Coll Cardiol 63:2346-2355

Publisher's Note Springer Nature remains neutral with regard to jurisdictional claims in published maps and institutional affiliations. 